\title{
Immunodeficiency viruses and prion disease
}

\author{
W. Bodemer \\ German Primate Center, Kellnerweg 4, 37077 Göttingen, Germany \\ Correspondence to: W. Bodemer (wbodemer@dpz.eu) \\ Received: 17 June 2015 - Revised: 30 July 2015 - Accepted: 4 August 2015 - Published: 24 August 2015
}

\begin{abstract}
Two threatening human diseases have emerged during the past 35 years. Human immunodeficiency virus (HIV) was transmitted from non-human primates - e.g., the chimpanzee to humans - and then spread into populations all over the world. To date, around 35 million people are infected and no vaccine is available because the virus undergoes rapid mutation, resulting in a swarm of virus strains. At best, therapeutical intervention is possible with antiviral drugs; however because of its capacity to rapidly mutate, resistant virus strains develop. Since non-human primates (NHPs) carry simian immunodeficiency virus (SIV), we could assess infection and immunity by SIV/HIV in rhesus monkeys ( $M$. mulatta) as a model for acquired immunodeficiency syndrome (AIDS).

Transmissible spongiform encephalopathy (TSE) emerged in ruminants in the 1980s and shortly thereafter appeared in humans, leading to variant Creutzfeldt-Jakob disease (vCJD). The vCJD is a terminal neurological disorder since it heavily and irreversibly damages the brain. No cure is at hand. The causative agents for TSE are prions. They are unusual pathogens and enigmatic since they lack nucleic acid as inheritable information. On the other hand, prions were suspected as infectious agents for years and suspected to be the etiological agent of scrapie in sheep. Molecular biology and medicine have clearly identified prions in recent years as the responsible agent for bovine spongiform encephalopathy in ruminants (BSE). BSE has been transmitted to humans, resulting in around $225 \mathrm{vCJD}$ cases. Similar to the SIV/HIV model for Acquired Immunodeficiency Syndrome (AIDS), we could establish a prion infection model in rhesus monkeys.

HIV/AIDS and vCJD are zoonoses since their original pathogens can be transmitted from animals to humans. Our experimental efforts to understand these intriguing pathogens and their corresponding diseases in rhesus monkeys as a valid model for both human diseases are summarized in this review.
\end{abstract}

\section{Research activities}

In July 1970 Hans-Jürg Kuhn submitted a memorandum entitled "Primatenforschung" which provided the basis for the planning of the German Primate Center (DPZ) as a multidisciplinary institute. One of the six proposed departments was "Virology and tissue culture". In 1983 Gerhard Hunsmann was appointed as head of the department virology. He was recognized as an expert not only for murine retroviruses but also for the emerging human T-cell leukemia virus (HTLV) and eventually human immunodeficiency virus (HIV). His experience from initial work at the former Max Planck Institute for Virology in Tübingen, Germany, and at the Tumor Institute in Freiburg im Breisgau, Germany, was a prerequisite for a vivid virology section at the DPZ. Consequently, retrovirology was a prominent research area at the DPZ from early on. In the beginning, serology to detect virus-specific antibodies was stepwise extended to studying immune response and attempts to conceive a vaccine against HIV. Even in those days, some scientist realized that the rhesus macaque may serve as the animal model for AIDS - not the least since non-human primates could be infected with human (HIV) as well as simian immunodeficiency virus (SIV). Infection, immunity, progression to disease and clinical symptoms resembled HIV-associated AIDS. This early scientific activity was seminal for ongoing work and to a certain degree still is today. Lessons learned by the immunodeficiency viruses may also be helpful in meeting new scientific approaches to fight Ebola in the future. 
In the 1990s HIV and AIDS dominated virology because the infection had spread around the world. Concepts to establish a vaccine relied on successful traditional vaccines like polio, measles and many more. However, first doubts emerged that the traditional vaccine strategies would not be applicable because HIV/SIV rapidly mutated and a HIV vaccine would most likely fail with respect to sterile immunity. This reminded us of the unsuccessful anti-lentiviral vaccines developed for horses against infection/disease by equine infectious anemia (EIAV). To illustrate how many strategies came up, the author's first report at the DPZ to join the virology department dealt with salmonella-gp160 HIV vectors as a mucosal vaccine. This vaccine has not been pursued since AIDS research recognized that protective immunity is not established by any vaccine directed against the complete gp160 envelope protein or parts thereof.

In addition, evidence accumulated that chimpanzees are not suitable as a HIV vaccine model. They could be infected but never progressed to disease. Nevertheless, molecular virology found the reason for this resistance against the virus, i.e., resistance, is brought about by epigenetic mechanisms, namely APOBEC deamination. APOBEC (apolipoprotein B mRNA editing enzyme, catalytic polypeptide-like) belongs to a family of functionally conserved proteins. Therefore, we could turn to macaques as a valid model at the DPZ, which already had a rhesus monkey colony. The possibility to use NHPs at the DPZ was highly appreciated by several research groups in Germany and Europe at that time. HIV and its close relative, SIV, were available for infection studies. National and international funding supported the work at the DPZ and embedded the virology unit in research cooperatives with high scientific capacity.

The department's HIV/SIV research went along with a closer look on the immunology. The most important detection was the TH1 and TH2 helper T-cell balance in rhesus monkeys. These findings prompted us and allowed to design vaccine molecules and vaccination programs aiming at a specific immune response, either cytotoxic (TH1) or humoral (TH2). It was not known which one would be superior to prevent or at least attenuate viral infection. Unfortunately, none of the cumbersome vaccine trails was successful, although partial contributions to protection could be assessed. Even in 2014, rhesus and cynomolgus monkeys served as animal models in innovative vaccination strategies like the elimination of proviral DNA (Andrieu et al., 2014).

Working on HIV prompted us to turn to novel expression and perhaps immunization vectors. Consequently, Semliki Forest virus had been transferred from the Karolinska Institute, Stockholm (Peter Liljeström), to the department of virology at the DPZ. Although we did not develop the SFV vector for HIV/AIDS, it became a most efficient tool in our experiments to study human prion proteins and eventually to develop monoclonal antibodies against the human prion protein. Of note, we were the first ones to use a prime-boost protocol for induction of an antibody response aiming at hybridoma cell lines producing monoclonal antibodies. The prime-boost protocol was also considered to become a novel strategy to immunize humans against HIV/AIDS - at first with the antigen or peptide only and in a second step with a viral vector like SFV or vaccinia virus expressing the antigen of interest. There were prime-boost vaccine trails in 20132014 , and there will perhaps be more in the years to come.

Besides virology and immunology we had an opportunity to focus on the manifestation of non-Hodgkin's Lymphoma (NHL) emerging under immunosuppression caused by the immunodeficiency virus. At the time when many animals were enrolled in vaccine studies, we observed tumors in some animals. The NHLs developed, and we could analyze the oncogenic cells and tissues. The cooperation with Franz-Josef Kaup, head of the pathology unit at the DPZ, was most fruitful, and with our skills in molecular biology we substantially broadened our knowledge on the NHL present in some HIV-infected animals. The molecular characteristics of the oncogenic tissue and cells could be analyzed in detail with subtractive libraries. This method was new and laborious but quite successful in cooperation with Vyacheslav Z. Tarantul from Moscow. Again, these studies confirmed how adequate our macaque HIV/AIDS model was. Several publications and the thesis of Horst Hanig are documents of this intriguing study. As therapy against HIV/AIDS, we tried to transfer autologous $\mathrm{T}$ cells of a cynomolgus monkey after the animal showed symptoms of S-AIDS. T cells were isolated and expanded in cell culture after immortalization with the herpesvirus saimiri. The New World monkey virus had been shown by our colleagues in Erlangen to immortalize human $\mathrm{T}$ cells (Helmut Fickenscher and Bernhard Fleckenstein). Thus larger amounts of $\mathrm{T}$ cells could be grown in culture. Most importantly, the virus-transformed T cells did not lose their immunological functions. Thus, these expanded $\mathrm{T}$ cells could be transferred back into the donor animal to reconstitute the decreasing T-cell population after the SIV infection. In one experiment we obtained evidence that the virus-transformed $\mathrm{T}$ cells were not malignant cells as we always suspected.

In the neighborhood of the DPZ, we cooperated with the neurologists at the University Hospital in Göttingen. Turning our attention to TSE allowed us to work with the CreutzfeldtJakob disease research group (Inga Zerr, Walter SchulzSchaeffer, Sigrid Poser, Klaus Felgenhauer and the group of Hans A. Kretzschmar). Weekly seminars were arranged; additionally, prion research novel strategies to develop viruslike particles (VLP) as a vaccine against neurodegenerative viral diseases were discussed, conceived and experimentally realized by Thomas Weber, Corinna Trebst and Wolfgang Lüke in the virology unit at the DPZ.

First steps into research on TSE or prions were taken in 1993/1994 - a time when BSE occurred in England but not all over Europe or globally. We became partner in a research cooperative headed by John Collinge at the MRC, London, and Hans A. Kretzschmar at the University Hospital in Göt- 
tingen. BSE was known from England, and it was suspected that a variant form of vCJD was caused by uptake of BSE prions. Since the spread of BSE over Europe or globally could not be excluded and therefore new human cases with vCJD might occur, research was intensified (Bodemer, 2009). Diagnostic tools to detect prions, namely the normal prion protein $(\mathrm{PrPc})$ or its disease-associated, abnormal prion protein isoforms (PrPd), was difficult since there were no antibodies available as molecular probes for immunohistochemistry or in ELISA (enzyme-linked immunosorbent assay) type assays. In a project funded by the German Federal Ministry of Education and Research (BMBF) we were able to raise immune response in mice and established a large number of hybridioma cell lines producing anti-PrP monoclonal antibodies. Quite in contrast to traditional immunization of mice and aiming at hybridomas secreting the antibody of interest, we have chosen a prime-boost protocol. This protocol started with immunization of mice with PrP coding DNA followed by a boost with SFV vectors carrying the identical PrP coding DNA. After this boost SFV transported the PrP sequence through the body and the final PrP molecule was presented to all those sites where the immune system could be triggered. The monoclonal antibodies (mabs) that were obtained proved to be perfect probes to detect PrP and its disease-associated variant PrP. These mabs provided a molecular tool to identify and define a certain functional region of the "folding-prone" PrP. Furthermore, some of the mabs were found to be sensitive probes to detect $\mathrm{PrP}$ in brain tissue from BSE-infected cattle. Of note, two commercially available BSE test kits with highest sensitivity, specificity and reproducibility relied on one of our mabs. The efficient procedure to generate these mabs was part of Susanne Krasemann's thesis. An example for the quality of the monoclonal antibodies in immunohistochemistry is shown in Fig. 1 with the mab 12F10. Brain and heart tissue was stained with $12 \mathrm{~F} 10$ and clearly showed levels of disease-associated PrP even in heart tissue. Beyond basic science on the structure of PrP molecules and generating versatile tools for PrP diagnostics, some mabs were commercialized and belonged to the best BSE test kits available. The latter was substantial to initiate new research programs and research groups at the DPZ. This story resembles the fate of the VLP a few years later.

Rhesus monkeys as a model for prion disease allowed circadian rhythm parameters to be monitored, which is not possible in human CJD cases. In parallel we proved transmission of BSE and human vCJD prions to a non-human primate species (Asante et al., 2015). This comprehensive project was funded by the EU and headed by John Collinge and Adriano Aguzzi working in London and Zurich, respectively. In fact, this project was supposed to be conducted in Vienna, Austria, but was for many reasons transferred to the DPZ by Franz-Josef Kaup in 2001. The project was unique since ethologists from the University of Vienna (Ivo Machatschke and John Dittami) had already joined the team and continued with their research at the DPZ. A dual approach was cho-
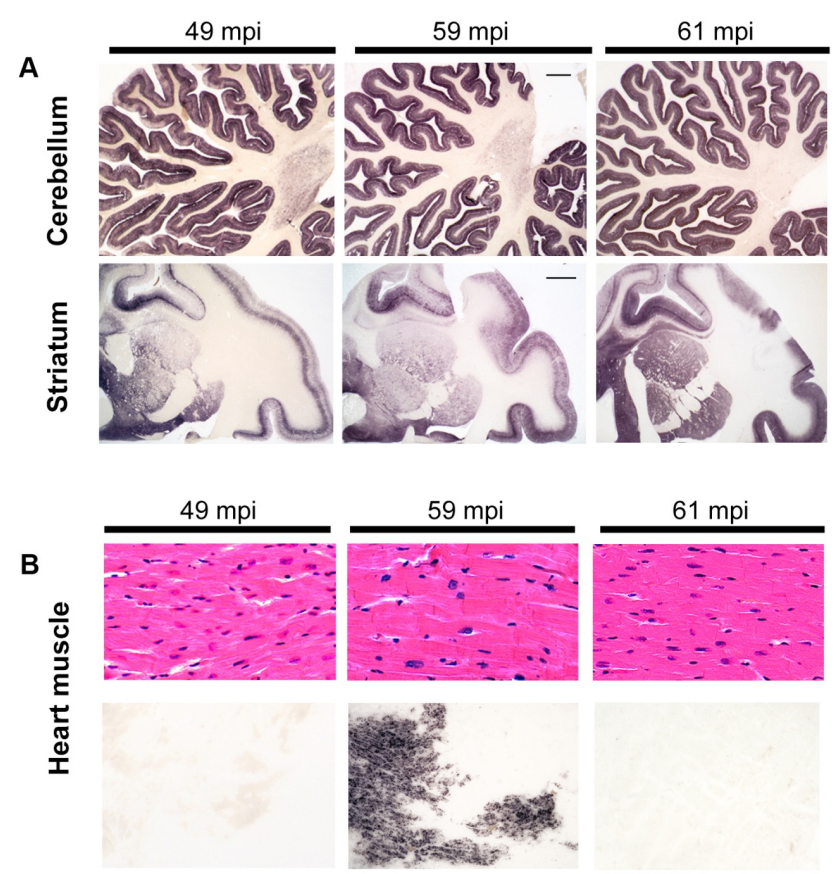

Figure 1. Tissue samples form three rhesus monkeys (49 mpi; $59 \mathrm{mpi} ; 61 \mathrm{mpi}$ ) infected with BSE prions and with clinical symptoms were analyzed with the monoclonal antibody (mab) 12F10. (a) Samples from brain (cerebellum and striatum). (b) Samples from heart muscle. With modifications from Krasemann et al. (2013).

sen, by first analyzing the behavior of the animals on a regular basis in order to notice the emergence and progress of the disease. In all, the course of the infection was observed over a period of about 5 years, from before inoculation until the emergence of behavioral symptoms. Secondly, via peritoneally implanted telemetry sensors, core body temperature and activity patterns could be tracked for almost 2 years. This study provided interesting results on behavioral and physiological changes that happened during the course of the prion diseases. However, telemetry did not reveal changes in sleepwake cycles, whereas significant pathological symptoms and behavior appeared only at late stages of the disease. The rather long time between infection and clinical symptoms also confirmed those "prion symptoms" seen in humans at late stages of variant vCJD. Years after early signs of prion infection appeared, most animals died from neurodegeneration, a clinical outcome known from human vCJD cases.

In order to identify prion-associated neuropathological changes, the distribution of proteinase-K-resistant prion protein (PrPres) was assessed by immunohistochemistry and protein/tissue blotting techniques. Besides brain we found several organs like heart and muscles with PrPd. Susanne Krasemann, a former PhD student in the virology department and now working with Markus Glatzel at the UKE, 
A

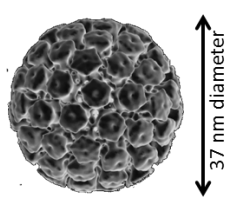

B

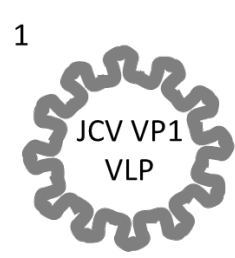

3

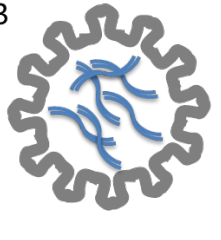

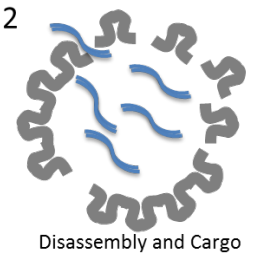

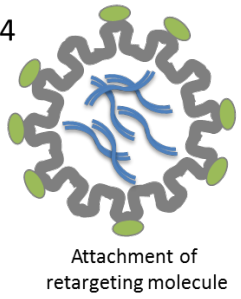

C HEK293-GFP (stable GFP expression)
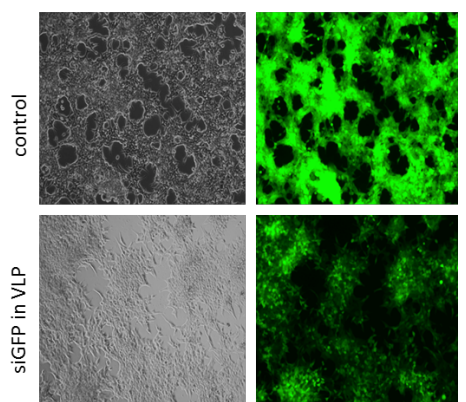

Figure 2. Virus-like particles (VLPs) of the human polyoma JC virus. Recombinant major capsid protein VP1 of the JC virus spontaneously forms VLPs. (a) 3-D reconstitution of electronmicrographs displaying the highly symmetric VLPs that are assembled from 72 pentameric capsomers. (b) A schematic overview of steps to utilize the VLPs for targeted delivery. Cargos are loaded after dissociation of the purified VLPs and reassembled to pack the load (e.g., DNA or synthetic RNA molecules). Target specificity can be achieved by attaching ligands to the outer VLP surface (step 4) to facilitate cell internalization via binding-exposed cell markers. Stably GFP expressing human HEK293 cells were treated with retargeted VLPs carrying either a non-cognate siRNA (control) or siRNAs targeting the GFP-mRNA (siGFP). A drastic reduction of the GFP expression can be seen after siGFP delivery in VLPs, while the cells treated with the control formulation remain unaffected and display homogenous and strong GFP expression. (c) Fluorescence assays to demonstrate targeted delivery of siRNA into HEK 293 cells.

Hamburg, made substantial contributions to this part of the EU-funded project.

Similar to our efforts to find a treatment against AIDS, we were considering how a therapeutic measure might be developed against prion infection, at least to slow down prion disease. With Philipp Schucht and Adrianoa Aguzzi we could deliver a PrP-carrying lentiviral vector into the brain of rhesus monkeys by neurosurgery. This therapeutic intervention was not successful since it was too late to turn down PrP syntheses in the brain. Nevertheless, it was proof of principle that a gene therapy might be feasible. Our animals offered many opportunities for further experiments. It was possible to derive cell lines from prion-infected monkeys. Sarah Tabrizi and John Collinge received tissue and succeeded to establish cell lines to analyze PrP. In the meantime, studies with non-human primates and prions are drastically reduced since infectivity of prions has been confirmed in several animal species. No promising therapeutic substance is available that would justify and require the rhesus monkey as a model.

Virus research and neurodegeneration were also looked at during our cooperation with neurologist in the University Hospital Göttingen, especially with Thomas Weber and Corinna Trebst. They considered virus-like particles (VLPs) based on a polyoma virus structural protein as a means to fight progressive multifocal leukoencephalopathy (PML). Expression vectors for particles were conceived, developed and then produced in the DPZ's virology department under supervision of Wolfgang Lüke - later on by Josef Metze and Karen Lemke at the Institut für Bioprozess und Analysemesstechnik (iba), Heiligenstadt, Thuringia. These days, VLPs are further developed as a biological shuttle to deliver regulatory nucleic acid species like miRNA - work that is being done in the recently established DPZ research group RNA Biology, headed by Jens Gruber, who also provided the structure of the polyoma-virus-derived VLP (Fig. 2).

\section{Conclusions}

To conclude, Hans-Jürg Kuhn once established a research place not only for service to study NHPs in zoological gardens or in the wild but also providing animals for intramural and external research groups to conduct their basic research. Whenever one talked to him about cooperations, the openminded Hans-Jürg Kuhn always supported such ideas and his "yes, go on" was granted. This open-mindedness led in the end to most exciting results with respect to HIV/AIDS 
and the model SIV/SAIDS. Although a vaccine could not be developed, the impact of $\mathrm{TH} 1$ or $\mathrm{TH} 2$ cells in the acquired immune response was new and offered, besides the virus's high mutation rate, an additional explanation of why vaccine strategies have failed so far. How NHLs develop was successfully analyzed and we were a partner of a Sonderforschungsbereich (SFB) at the University Hospital Göttingen, headed at that time by Wolfgang Hiddemann. Prion research in national and international research programs took place over many years. Monoclonal antibodies "invented" at the DPZ entered BSE diagnostics. Introducing new vector system like Semliki Forest virus or the polyomavirus-derived VLP broadened our expertise in virology. Recently and thanks to our rhesus monkey model, we could turn to study epigenetics - studies that would not have been possible in human CJD or VCJD cases. Brain tissue could be assessed for the presence and type of non-coding small RNA species like the Alu and BC200 transcripts. Epigenetic modification like deamination was revealed for the first time. This panel of attractive research areas states how important Hans-Jürg Kuhn was for the DPZ in Göttingen.

Edited by: E. Fuchs

Reviewed by: two anonymous referees

\section{References}

Andrieu, J. M., Chen, S., Lai, C., Guo, W., and Lu, W.: Mucosal SIV vaccines comprising inactivated virus particles and bacterial adjuvants induce CD8(+) T-regulatory cells that suppress SIV-positive CD4(+) T-cell activation and prevent SIV infection in the Macaque model, Front Immunol., 5, 297, doi:10.3389/fimmu.2014.00297, 2014.

Asante, E. A., Smidak, M., Grimshaw, A., Houghton, R., Tomlinson, A., Jeelani, A., Jakubcova, T., Hamdan, S., Richard-Londt, A., Linehan, J. M., Brandner, S., Alpers, M., Whitfield, J., Mead, S., Wadsworth, J. D., and Collinge, J.: A naturally occurring variant of the human prion protein completely prevents prion disease, Nature, 522, 478-481, doi:10.1038/nature14510, 2015.

Bodemer, W.: Prions, in: Encyclopedia of Microbiology 3rd Edn., edited by: Schaechter, M., Academic Press, 689-706, 2009.

Krasemann, S., Mearini, G., Krämer, E., Wagenführ, K., SchulzSchaeffer, W., Neumann, M., Bodemer, W., Kaup, F. J., Beekes, M., Carrier, L., Aguzzi, A., and Glatzel, M.: BSE-associated prion-amyloid cardiomyopathy in primates, Emerg Infect Dis., 19, 985-988, doi:10.3201/eid1906.120906, 2013. 